\title{
EXTRACTION OF LEAF ANGLE DISTRIBUTION FROM AN INDIVIDUAL BROADLEAF TREE USING TERRESTRIAL LASER SCANNING DATA
}

\author{
Yiming Chen ${ }^{1}$ *, Zhengjun Liu ${ }^{1}$, Wuming Zhang ${ }^{2}$, Chen Qiao ${ }^{3}$, Haiyan $\mathrm{Gu}^{1}$ \\ ${ }^{1}$ Chinese Academy of Surveying \& Mapping, Beijing 100036, China - (chenym, zjliu)@ casm.ac.cn \\ ${ }^{2}$ Institute of Remote Sensing Science and Engineering, Faculty of Geographical Science, Beijing Normal University, Beijing 100875 , \\ China - wumingz@bnu.edu.cn \\ ${ }^{3}$ Department of Earth System Science, Tsinghua University, Beijing 100084, China - qiaochenbnu@ gmail.com
}

Commission VI, WG VI/4

KEY WORDS: Leaf angle distribution, Terrestrial laser scanning, Leaf segmentation, Leaf area index, point cloud

\begin{abstract}
:
The angular distribution of leaves is a key vegetation structural parameter for evaluating the reflection and transmission of solar radiation through vegetation canopies. Accurate extraction of Leaf Angle Distribution (LAD) is of great importance in estimating other vegetation structural parameters such as the canopy clumping and leaf area index. However, field measurement of LAD is timeconsuming, labour-intensive and subjective. In most studies, LAD is assumed to follow the spherical distribution assumption within canopy which may lead to considerable errors. To address this issue, we proposed a new approach for leaf segmentation and LAD measurement of individual broadleaf tree based on the TLS point cloud data. Based on the point density, point continuity and the distribution of intensity in the point cloud, this approach provides a fast and accurate leaf segmentation and LAD extraction strategy. Results of this TLS-based LAD method compared well with that extracted by the field measurement and the MDI-based method. This strategy shows its potential and applicability in accurate LAD measurement and LAI estimation.
\end{abstract}

\section{INTRODUCTION}

The angular distribution of leaves is a key vegetation structural parameter for evaluating the reflection and transmission of solar radiation through vegetation canopies. Accurate extraction of Leaf Angle Distribution (LAD) is of great importance in estimating other vegetation structural parameters such as the canopy clumping and leaf area index. However, the leaf angle distribution showed its seasonal and interannual variation and changed with height within canopies (Kull et al., 1999, Raabe et al., 2015). It is difficult to measure LAD in a fast and accurate way. In the past decades, researchers have proposed many approaches for the field measurement of LAD which can be categorized as direct measurements and indirect measurements.

Direct methods measure the LAD manually with the help of compass and protractor (Daughtry, 1990; Ross, 2012). The compass is used to measure the leaf azimuth angle. The protractor or the clinometer is used for measuring leaf zenith angle manually. These methods are labour-intensive, timeconsuming and destructive in the field measurement. In addition, the leaves of tall trees are hard to access. Instead of the measurements using compass and protractor, some researchers proposed the 3-D digitalization measurement. (Lang, 1973) designed a digitalized 3-D coordinate system for the angle measurement of each single leaf in the 3-D space. (Sinoquet et al., 1998) improved the 3-D digitalization technology and build an electromagnetic digitizer which can successfully build a 3-D model of the entire plant canopy and measure the LAD. However, these methods take a long time in acquiring data and failed to respond to the dynamic change of LAD in a short time.
In order to measure the $\mathrm{LAD}$ in a larger scale and overcome the time-consuming in manually measurement, indirect methods were proposed. Indirect LAD measurements include the LAD retrieval method based on the gap fraction model, digital photogrammetry method, and 3-D reconstruction method.

The theoretical basis of the retrieval methods is the Beer's Law. By acquiring the penetration of light radiation through canopies, the LAD information can be retrieved based on the Beer's Law and the canopy gap fraction model (Chen et al., 1997; Li et al., 1997). Many instruments used these methods to acquire the gap fraction and retrieve the Leaf Area Index (LAI) and average leaf inclination angle, such as TRAC, Hemiview and LAI-2000. However, the inclination angle of each leaf cannot be extracted. In addition, the use of these optical instruments was limited by the illumination conditions.

Another indirect method based on the digital photogrammetry to calculate the leaf angle through digital photos (Ryu et al., 2010). This method selected leaves oriented approximately parallel to the viewing direction of the digital camera and measure LAD using the angle measurement tool of a public domain image processing software. This method offers a simple, reliable and reproducible way for the LAD extraction and has been widely used. (Zou et al., 2014) has successfully introduced this method in the LAD measurement of crops. Moreover, (Mcneil et al., 2016) fixed a digital camera to the platform of unmanned aerial vehicle (UAV) and measured the LAD in a larger scale based on this method. However, in this method, the angle of an individual leaf can only be extracted if the leaf oriented parallel to the viewing direction.

\footnotetext{
* Corresponding author
} 
The 3-D reconstruction method for measuring LAD used the digital photos taken from multiple angles and the photogrammetry theory for 3-D reconstruction and angle measurement of each leaf (Wang et al., 2009, Muller-Linow et al., 2015). This method is a non-destructive, low-cost and objective way for the LAD measurement.

In recent years, Terrestrial Laser Scanning (TLS) has been widely used in extracting forest structural parameters (Zhao et al., 2015; Hosoi et al., 2015). TLS is an active technology which acquires the finest 3-D characteristics of canopy structure and offers a new strategy for the ground-based measurement of LAD.

In this paper, we propose a new approach for leaf segmentation and LAD measurement of an individual broadleaf tree based on the TLS data. Based on the point density, point continuity and the distribution of intensity in the point cloud, this approach provides a fast and accurate leaf segmentation strategy for LAD measurement. To evaluate our method, we compared the LAD results to the manual measurement of an artificial tree indoor. As the manual measurement of LAD was impractical in the field we applied the Multi-Directional Imager (MDI) to acquire digital photos from multiple views and extracted the LAD of a broadleaf tree for evaluation. Results showed that the TLSbased LAD compared well with both the manual measured and MDI-based LAD.

\section{METHODOLOGY}

The TLS point cloud of an individual tree include the branch points, stem points and leaf points. To reconstruct the leaf surface and extract the accurate leaf inclination, the points of each single leaf need to be segmented first. Therefore, the workflow of this TLS-based LAD extraction strategy is shown as follows:

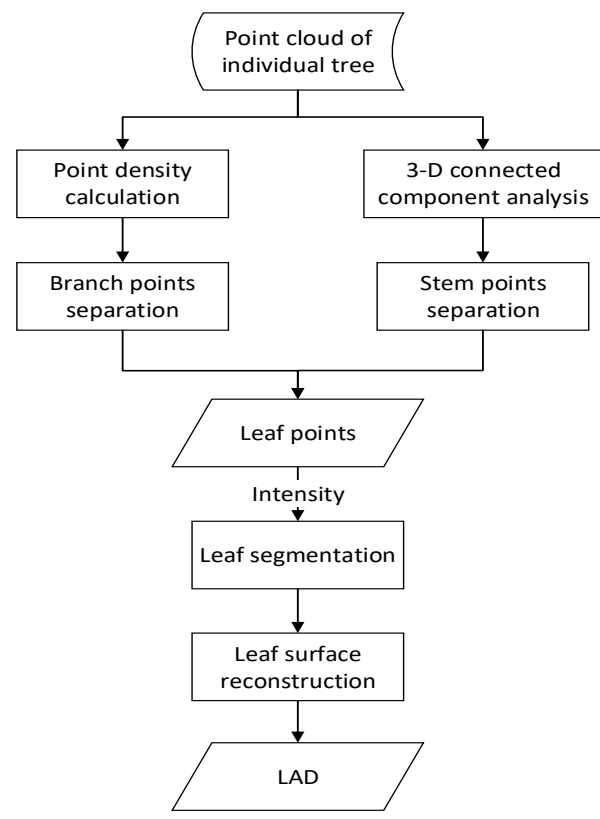

Figure 1. Workflow of the proposed TLS-based LAD extraction strategy

\subsection{Branch Points Separation}

In this study, the average leaf width of broadleaf tree was approximately $4 \mathrm{~cm}$. However, the diameters of branches were smaller than $2 \mathrm{~cm}$ except for the primary branches. Therefore, the amount of leaf points was obviously larger than that of branch points within the same 3-D neighbourhood space as shown in Figure 2.

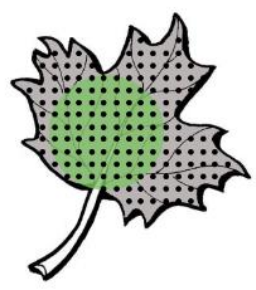

(a)

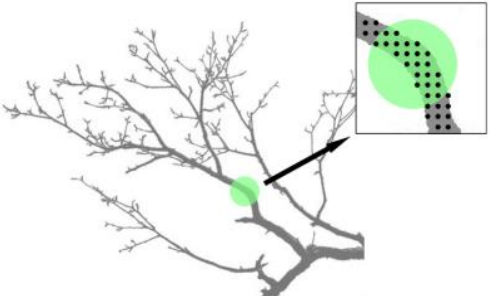

(b)
Figure 2. Sketch map of the amount of leaf points (a) and branch points (b) within the same neighbourhood space (the green area)

In this study, the size of the neighbourhood space was determined by the leaf size. Thus, the branch points can be separated successfully using a proper threshold of the amount of 3-D neighbour points.

\subsection{Stem Points Separation}

In order to get the leaf points for the LAD extraction, the stem points and the primary branch points also need to be separated. Considering the spatial continuity of the stem and primary branch in the 3-D space, we applied the 3-D connected component analysis approach for the stem points separation. Given the minimum threshold of the connected points, stem and primary branch points were removed from the original point cloud.

\subsection{Leaf Segmentation}

After removal of the branch points and stem points, the leaf points were left in the point cloud. For further extraction of the angle of each leaf, leaf segmentation approach was proposed. As the beam size of each emitted laser was much smaller than the leaf width in the TLS data, the phenomenon of partial hits occurs when the laser falls on the edges of leaves (Figure 3(a)). Therefore, the intensity of the return pulse of the edge points was smaller than that of the interior leaf points (Figure 3(b)). Thus, each individual leaf was segmented with the help of the intensity information of each 3-D point.

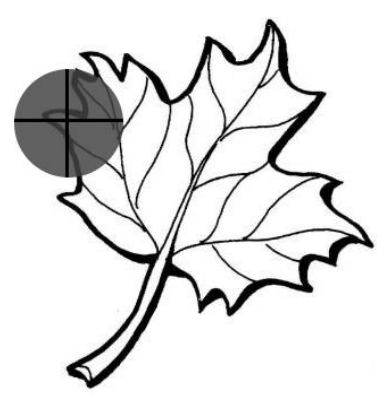

(a)

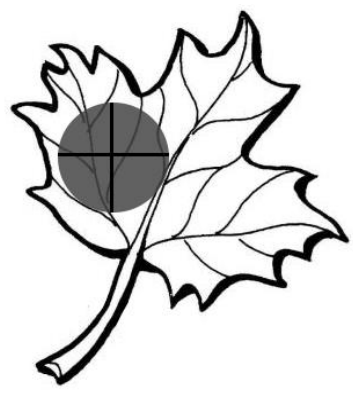

(b)
Figure 3. Sketch map of the position relationship between laser beam and single leaf 


\subsection{Leaf Surface Reconstruction and LAD Extraction}

Acquiring the points of each individual points, leaf surface needs to be fitted and reconstructed for the calculation of leaf inclination angle. In this study, as the curvature of the leaf is small, we used the least-square plane fitting approach for leaf surface reconstruction.

Then the normal vector of each leaf was computed and the leaf inclination angle was calculated as follows:

$$
\theta_{l}= \begin{cases}\arccos \left(\frac{n_{z}}{\|\vec{n}\|}\right) & \vec{n} \cdot \vec{v}>0 \\ 180^{\circ}-\arccos \left(\frac{n_{z}}{\|\vec{n}\|}\right) & \vec{n} \cdot \vec{v} \leq 0\end{cases}
$$

Where $\theta_{l}$ is the leaf inclination angle of leaf $l, \vec{n}=\left(n_{x}, n_{y}, n_{z}\right)$ is the normal vector of leaf $l$ in the 3-D space, and $\vec{v}$ is the vertical vector.

Finally, the LAD of the entire broadleaf tree can be extracted.

\section{EXPERIMENTS AND RESULTS}

\subsection{Artificial tree}

As described above, manually measurement of LAD from an individual tree is time-consuming and labour-intensive. In addition, the leaves on tall trees were unaccessible. Therefore, for the evaluation purpose, we set up an artificial broadleaf tree (Figure 4) indoor and measured the LAD using protractor.

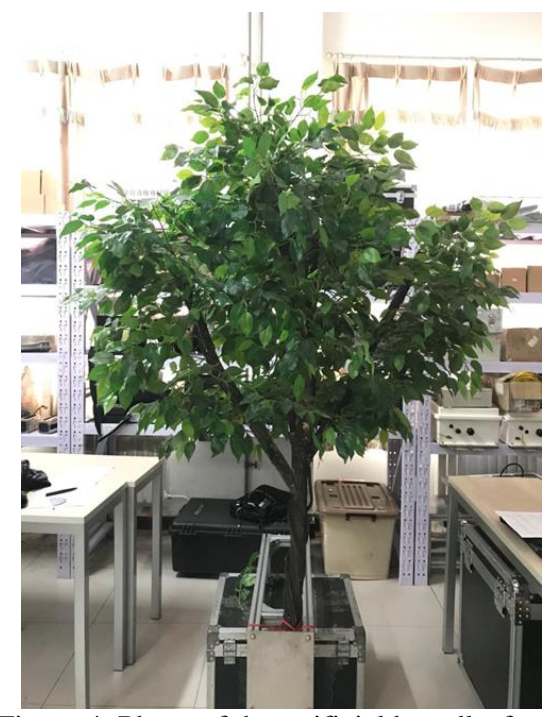

Figure 4. Photo of the artificial broadleaf tree

The height of the artificial tree is $2 \mathrm{~m}$. The size of each leaf is approximately $7 \mathrm{~cm} \times 4 \mathrm{~cm}$. In this study, we measured leaf inclination angle of 360 leaves in total. The average inclination angle was $53.22^{\circ}$. Figure 5 shows the LAD histogram with manual measurement.

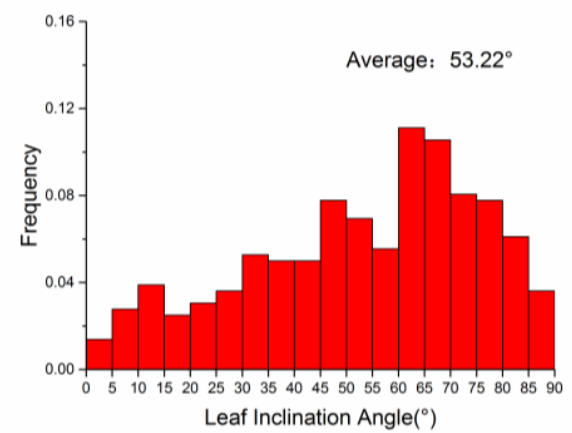

Figure 5. LAD histogram of the artificial tree with manual measurement

The TLS point cloud of this artificial tree was acquired by Riegl VZ-1000 instrument at the height of $1 \mathrm{~m}$. The horizontal distance between the tree and the laser scanner was $5 \mathrm{~m}$. The angle stepwidth was set as $0.03^{\circ}$ and other parameters of the scanner was shown in Table 1 . The original point cloud is shown in Figure 6.

\begin{tabular}{|c|c|}
\hline Sepcifications & Riegl VZ-1000 \\
\hline Beam divergence & $0.3 \mathrm{mrad}$ \\
Wavelength & $1550 \mathrm{~nm}$ \\
Beam diameter & $7 \mathrm{~mm}$ \\
Minimum range & $2.5 \mathrm{~m}$ \\
Maximum range & $350 \mathrm{~m}$ \\
Zenith angle & $30^{\circ}$ to $130^{\circ}$ \\
Azimuth angle & 0 to $360^{\circ}$ \\
\hline
\end{tabular}

Table 1. Riegl VZ-1000 specifications

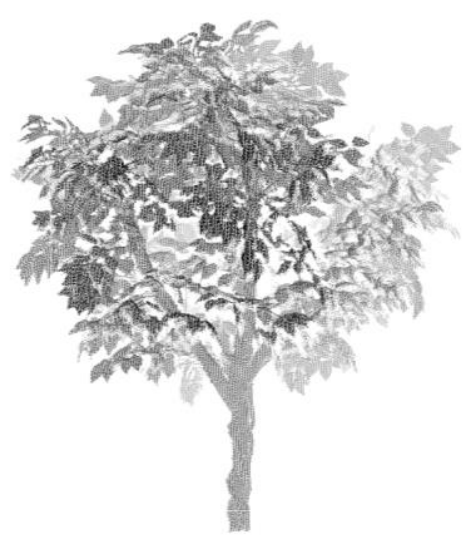

Figure 6. Point cloud of the artificial tree

The results of the branch points separation and stem points separation were shown in Figure 7(a) and 7(b) respectively. Then 165 leaves were segmented from the point cloud and the LAD histogram was shown in Figure 8. 


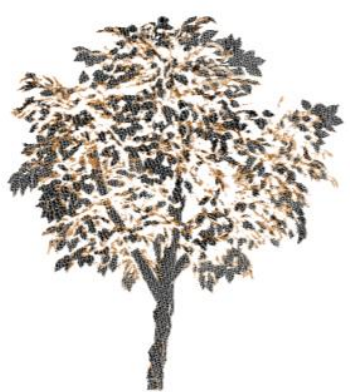

(a)

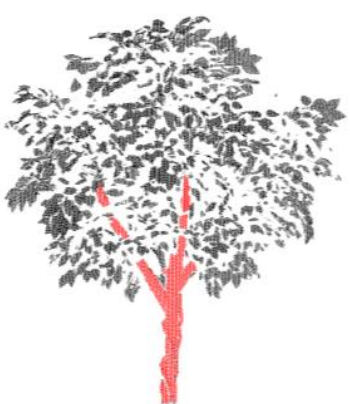

(b)
Figure 7. Results of the branch points separation (a) and stem points separation (b)

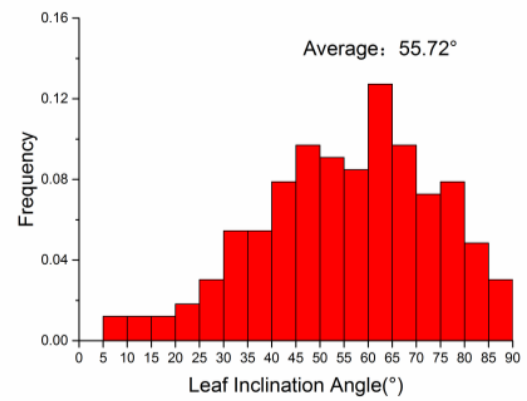

Figure 8. TLS-based LAD histogram of the artificial tree

According to the results shown in Figure 5 and Figure 8, the TLS-based LAD compared well with the manually measured LAD. In addition, the average leaf inclination angles calculated were also comparable. The difference was mainly caused by two reasons: 1) The leaves inside the tree crown were occluded in the point cloud. Therefore, the amount of the leaves calculated with TLS measurement was smaller than that calculated with manually measurement. 2) The manually measurement of LAD was subjective to some extent which may lead to errors of the results.

\subsection{Actual broadleaf tree}

In the actual forest, it is hard to measure the LAD of an individual tree manually. For evaluation purpose, we used the MDI instrument which consists of tree simultaneous exposure cameras to acquire multi-view photos of a broadleaf tree as shown in Figure 9. Then the point cloud of the tree were then acquired using a dense matching algorithm. With the help of the open source software CloudCompare, we manually segmented 96 leaves and extracted the LAD. The histogram was shown in Figure 10.

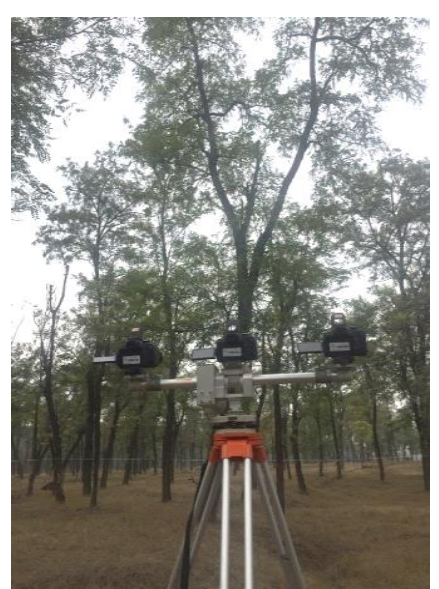

Figure 9. Photo acquisition using MDI instrument

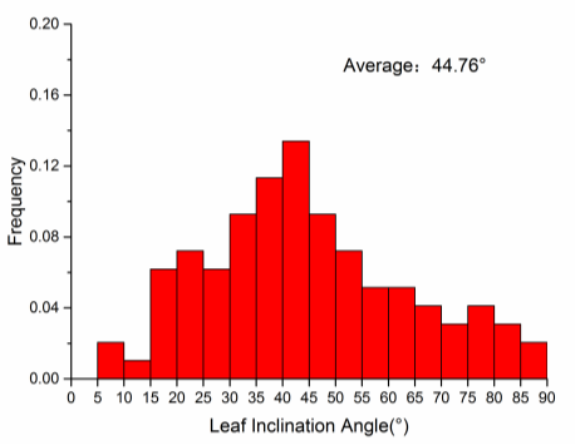

Figure 10. MDI-based LAD histogram of the actual tree

In this study, Riegl VZ-1000 was used for the acquisition of the TLS point cloud and the parameters were the same as used in Chapter 3.1. Figure 11 showed the point cloud of the broadleaf tree.

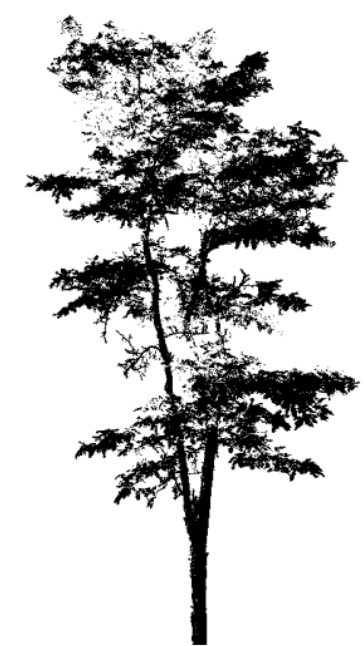

Figure 11. Point cloud of the actual broadleaf tree

The results of the branch and stem points separation were shown in Figure 12. The LAD histogram of this broadleaf tree calculated with our TLS-based method was then shown in Figure 13. 


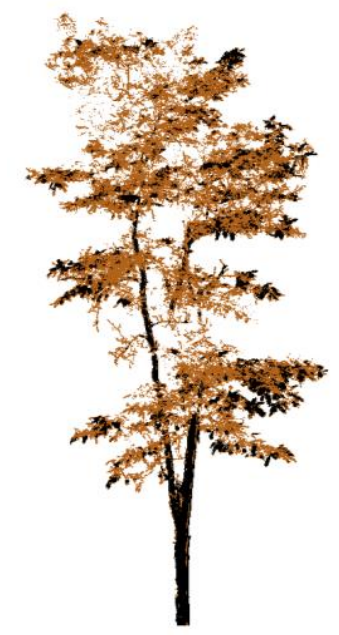

Figure 12. Results of branch and stem separation

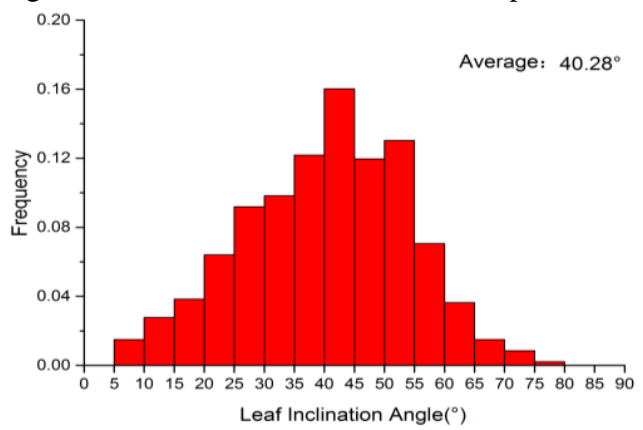

Figure 13. TLS-based LAD histogram of the actual tree

According to the results shown in Figure 10 and Figure 13, the TLS-based LAD compared well with the MDI-based LAD. The average leaf inclination angles calculated were also comparable with the absolute error of $4.48^{\circ}$. The difference was mainly caused by the quality of the photos acquired by the MDI instrument and the quality of the dense matching algorithm.

\section{CONCLUSIONS}

In this paper, we proposed a new strategy for leaf segmentation and LAD extraction based on the point cloud of the individual tree acquired with TLS. Based on the point density in the 3-D space and the point continuity in the point cloud, this method separated the point cloud into branch points, stem points and leaf points. Using the distribution of intensity within single leaf, the points of each leaf was segmented and the inclination angle was calculated to compute the LAD. This study provides a method for measuring actual LAD automatically and accurately using the TLS-based point cloud. The results of the LAD using this TLS-based method compared well with that using the field measurement and the MDI-based method. This TLS-based method shows its potential and applicability in accurate LAD measurement and LAI estimation.

\section{ACKNOWLEDGEMENTS}

This work is supported by funds from CASM No. AR1915. This work was also supported by the National Key R\&D Program of China Grant No. 2016YFB0501400 and 2013 CB733402.

\section{REFERENCES}

Kull, O., Broadmeadow, M., Kruijt, B., Meir, P., 1999. Light distribution and foliage structure in an oak canopy. Trees, 14(2), 55-64.

Raabe, K., Pisek, J., Sonnentag, O., Annuk, K., 2015.

Variations of leaf inclination angle distribution with height over the growing season and light exposure for eight broadleaf tree species. Agricultural and Forest Meteorology, 214, 2-11.

Daughtry, C.S., 1990. Direct measurements of canopy structure. Remote Sensing Reviews, 5(1), 45-60.

Ross, J., 2012. The radiation regime and architecture of plant stands. Springer Science \& Business Media.

Lang, A., 1973. Leaf orientation of a cotton plant. Agricultural Meteorology, 11, 37-51.

Sinoquet, H., Thanisawanyangkura, S., Mabrouk, H., Kasemsap, P., 1998. Characterization of the light environment in canopies using 3D digitising and image processing. Annals of Botany, 82(2), 203-212.

Chen, J.M., Rich, P.M., Gower, S.T., Norman, J.M., Plummer, S., 1997. Leaf area index of boreal forests: theory, techniques, and measurements. Journal of Geophysical Research: Atmospheres, 102(D24), 29429-29443.

Li, X., Wang, J., Xiang, Y., 1997. Measuring leaf angle distribution and leaf area index of conifer canopies simultaneously on wide angle image. Journal of Remote Sensing, 1, 54-61.

Ryu, Y., Sonnentag, O., Nilson, T., Vargas, R., Kobayashi, H., Wenk, R., Baldocchi, D.D., 2010. How to quantify tree leaf area index in an open savannah ecosystem: a multi-instrument and multi-model approach. Agricultural and Forest Meteorology, 150(1), 63-76.

Zou, X., Mõttus, M., Tammeorg, P., Torres, C.L., Takala, T., Pisek, J., Mäkelä, P., Stoddard, F.L., Pellikka, P., 2014. Photographic measurement of leaf angles in field crops. Agricultural and forest meteorology, 184, 137-146.

Mcneil, B.E., Pisek, J., Lepisk, H., Evelin, H., 2016. Measuring leaf angle distribution in broadleaf canopies using UAVs. Agricultural and forest meteorology, 218, 204-208.

Wang, H., Zhang, W., Zhou, G., Yan, G., Clinton, N., 2009. Image-based 3D corn reconstruction for retrieval of geometrical structural parameters. International Journal of Remote Sensing, 30(20), 5505-5513.

Müller-Linow, M., Pinto-Espinosa, F., Scharr, H., Rascher, U., 2015. The leaf angle distribution of natural plant populations: assessing the canopy with a novel software tool. Plant methods, 11(1), 11 .

Zhao, K., García, M., Liu, S., Guo, Q., Chen, G., Zhang, X., Zhou, Y., Meng, X., 2015. Terrestrial lidar remote sensing of 
forests: Maximum likelihood estimates of canopy profile, leaf area index, and leaf angle distribution. Agricultural and Forest Meteorology, 209, 100-113.

Hosoi, F., Omasa, K., 2015. Estimating leaf inclination angle distribution of broad-leaved trees in each part of the canopies by a high-resolution portable scanning lidar. Journal of Agricultural Meteorology, 71(2), 136-141. 